\begin{tabular}{c} 
Volume and Issues Obtainable at Center for Sustainability Research and Consultancy \\
Journal of Business and Social Review in Emerging Economies \\
ISSN: 2519-089X (E): 2519-0326 \\
Volume 6: No. 1, March 2020 \\
CSRᄃ \\
Journal homepage: www.publishing.globalcsrc.org/jbsee \\
\hline
\end{tabular}

\title{
Relationship between Physical Activity and Burnout among University Faculty in Pakistan
}

\section{${ }^{1}$ Asif Ali, ${ }^{2}$ Asif Naveed Ranjha, ${ }^{3}$ Syed Mussawar Hussain Bukhari}

${ }^{1}$ Associate Professor, Department of Physical Education \& Sports Science, Government College University, Lahore, Pakistan. Email: goraya128@ hotmail.com

${ }^{2}$ Associate Professor, Department of Social Work, The Islamia University of Bahawalpur, Pakistan: aasif_ranjha@yahoo.com

${ }^{3}$ Chairman, Department of Political Science, The Islamia University of Bahawalpur, Pakistan

\begin{tabular}{ll}
\hline \multicolumn{1}{c}{ ARTICLE DETAILS } & ABSTRACT \\
\hline $\begin{array}{l}\text { Hevistory } \\
\text { Available Online: } \text { March } 2020\end{array}$ & $\begin{array}{l}\text { Burnout can lead towards challenge in social interaction and } \\
\text { physical ailments. This study sought to determine the relationship of } \\
\text { physical activity with faculty burnout. A total of 254 faculty } \\
\text { members were surveyed from three public sector universities of }\end{array}$ \\
\hline Keywords & southern Punjab region of Pakistan. Physical activity and burnout \\
Burnout, Physical Activity, & were assessed using IPAQ short version and OLBI, respectively. \\
Faculty, University, Social & Findings indicated a significantly negative relationship of total \\
& score of physical activity with disengagement, exhaustion, and total \\
JEL Classification: & burnout score. Physical activity level (low, medium, high) was \\
D9, Z2 & sugnificantly negatively correlated with total burnout score and \\
& between total physical activity and burnout after controlling for age, \\
gender, experience, and academic qualification in partial correlation \\
analysis. In addition, it was observed that burnout increased and \\
physical activity decreased with increased age and job experience. \\
These findings suggest that the interventions dealing with faculty \\
burnout may consider physical exercise as a priority to prevent \\
faculty burnout. More priority needs to be given to the faculty \\
members with higher age and job experience.
\end{tabular}

OPEN ACCESS

(C) 2020 The authors, under a Creative Commons AttributionNonCommercial 4.0

Corresponding author's email address: aasif_ranjha@yahoo.com

Recommended citation: Ali, A., Ranjha, A. N. \& Bukhari, S. M. H., (2019). Relationship between Physical Activity and Burnout among University Faculty in Pakistan. Journal of Business and Social Review in Emerging Economies, 6(1), 1-8

DOI: $10.26710 /$ jbsee.v6i1.1021

\section{Introduction}

There is no consensus on the conceptualization of faculty burnout among researchers. In general, it can be defined as the responses to stress experienced in relation to university teaching that is generally characterized by experiencing excessive fatigue resulted from university job, cynical behavior towards 
university, feelings of insufficiency towards teaching related accomplishment (Golub, Johns III, Weiss, Ramesh, \& Ossoff, 2008; Washington*, Quanstrom, Carroll, \& Greene, 2019). Burnout has been linked with various negative consequences, such as decreased academic performance, poor physical health and depression (Sabagh, Hall, \& Saroyan, 2018), and inappropriate behaviors (Dyrbye et al., 2010). Evidence found existence of burnout among university professors (Lackritz, 2004), faculty physicians (Wright, Khetani, \& Stephens, 2011), university online teachers (Hogan \& McKnight, 2007), college professor (Gonzalez \& Bernard, 2006), pharmacy faculty (El-Ibiary, Yam, \& Lee, 2017), and in medical school faculty (Ahmady, Changiz, Masiello, \& Brommels, 2007). High burnout is also linked with high social anxiety (Koutsimani, Anthony, \& Georganta, 2019). Interventions contributing alleviation of faculty burnout and developing the opposite direction, is crucially important.

Even though conditions of faculty burnout hinder optimal functioning of physical and mental abilities, there is lack of evidences about the mechanisms underpinning its effects, or the conditions which aggravate burnout in university faculty. Physical activity can be one candidate that might contribute to understand burnout effects. Physical activity has been shown to improve physical, psychological, social, and personal functioning (Penedo \& Dahn, 2005). Physical activity found to be inversely related with job related exhaustion and absenteeism, and positively associated with job related attrition intentions (Carson, Baumgartner, Matthews, \& Tsouloupas, 2010). Toker and Biron (2012) indicated that individuals with higher level of physical activity exhibited less symptoms of job burnout and low level of depressive symptoms compared with low active individuals. These findings suggest that physical activity might work as buffering mechanism to reduce faculty burnout. There is lack of empirical data about the relationship between physical activity and burnout among university faculty.

More importantly, certain level of involvement in physical activity considered essential for physical and psychological health, and better quality of life. For adults, recent research has emphasized that adults should engage in 150 to 300 minutes per week in the physical activity of moderate intensity or vigorous physical activity for the duration of 50-75 minutes per week, or combination of both intensities of exercise along with to weight training related physical activity twice a week (Piercy et al., 2018; Singh, Pattisapu, \& Emery, 2019). According to the world health organization, in 2016, approximately 32 percent of women and 23 percent of men reported to be involvement in insufficient level of physical activity worldwide (Organization, 2019). Health problems including excessive fat, diabetes, increased health related cost, and increased chronic diseases are reported to associated with decreased physical activity (González, Fuentes, \& Márquez, 2017).

Even though, physical activity and faculty burnout seems distinct concepts, however, some researchers remain very curious concerning exploring links between these two variables. It appeared that physical activity thought to be positively related with various positive mental and psychological factors. In contrast, symptoms of burnout have been associated with various negative mental and psychological states. It logically leads to assume that there might be inverse relationship between these two concepts and physical activity might have buffering effect on faculty burnout.

Keeping in view the importance of physical activity with respect to physical and mental health, some researchers have been trying to understand the relationship between physical activity and burnout. There is paucity of research examined the relationship of physical activity and faculty burnout. For example, Sane, Devin, Jafari, and Zohoorian (2012) examined relationship between physical activity and burnout among university teachers. Results demonstrated a significantly negative association between the both variables suggesting effectiveness of physical activity to reduced symptoms of burnout among university faculty. Similarly, Moueleu Ngalagou et al. (2019) also reported significantly inverse relationship between physical activity and burnout among university teachers in Gameroon. Even though it has been demonstrated that burnout negatively related with cognition (May, Bauer, \& Fincham, 2015), emotions(Dyrbye et al., 2011), physiological functions (May, Sanchez-Gonzalez, Brown, Koutnik, \& Fincham, 2014) and academic performance (Herman, Hickmon-Rosa, \& Reinke, 2018), there is scarce of 
studies investigated the strategies or factors exerts positive effects on burnout among university faculty. The purpose of this study is to determine the association between physical activity and burnout among university faculty members in Pakistan. We expect that there will be negative correlation between these two variables in the sample used in this study.

\section{Methods}

\subsection{Participants}

A total of 287 faculty members with their ages ranged from 24 years to 60 years (age mean=36.56 years; $\mathrm{SD}=6.24$ ) having the average experience of 7.28 years were randomly selected out of 607 faculty members from three public sector universities of Sothern Punjab, Pakistan. Thirty three participants left the major portion of questionnaire incomplete or did not return the questionnaire to the investigator and were excluded from final analysis. Thus, the final sample size composed of 254 participants. Wide majority of the sample consisted of lecturers $(34.3 \%)$ and assistant professors $(63.8 \%)$. The proportion of associate professor and professors in selected sample was $1.2 \%$ and $.8 \%$, respectively. While, $62.2 \%$ were male and $37.8 \%$ were female faculty members. More than half of the participants had PhD Degree $(52.4 \%)$ whereas the academic qualification of $40.2 \%$ and $7.4 \%$ was MPhil and Master Degrees, respectively. $79.92 \%$ faculty members had less than ten years of experience and only $20.07 \%$ participants had the experience above than ten years. All participants voluntarily took part in the study. The anonymity and confidentiality of the information obtained by the participants were insured. All data was collected during October and November, 2019.

\subsection{Measures}

The participants were asked to complete a questionnaire consisting of three sections such as demographics, physical activity and burnout.

\subsubsection{Demographics}

In this section, participants responded to the questions concerning name of university, department, designation, age, gender, education level, and teaching experience.

\subsubsection{Physical activity}

The short version of international physical activity questionnaire that consists of seven items was administered to assess the level of physical activity in the respondents. This tool has sufficient level of validity and reliability to quantify physical activity in many countries including Pakistan and also has been consistently used in previous research (Craig et al., 2003; Samir, Mahmud, \& Khuwaja, 2011). First six items of this tool requires the participants to report frequency and duration involving in low, moderate and vigorous physical activity during the previous week. The seventh item quantifies the total sedentary time during the week days. For the purpose of analysis, the metabolic equivalent task (MET) weeks/minutes was calculated from the raw data obtained through six items according to the guideline of IPAQ scoring procedure (Committee, 2005). Whereas, the data of seventh item was used separately in the analysis. Based on total MET, participants were further categorized as low, medium and high active individuals according to the guidelines of this tool.

\subsubsection{Burnout}

Burnout of faculty members quantified by administering The Oldenburg Burnout Inventory (OLBI) consisted of 16 items and two subscales including disengagement and emotional exhaustion (Demerouti, 1999; Demerouti \& Nachreiner, 1998). Each of the two factors consisted of eight items such as "I always find new and interesting aspects in my work" to assess disengagement and questions such as "There are days when I feel tired before I arrive at work" to assess exhaustion. Four responses from strongly agree to strongly disagree were presented against each question. Scoring of the Items with negative wording was reversed and scoring was arranged such that higher score represented greater disengagement or exhaustion. This tool considered robust with respect to reliability and validity to assess burnout in academic and job setting (Reis, Xanthopoulou, \& Tsaousis, 2015). Both subscales were separately 
included in analysis. In addition, burnout was defined by achieving the score from summing up the score of these two subscales. The scores of both subscales and total burnout were included in analysis.

\section{Analysis}

Descriptive statistics was used to calculated frequencies of the data. In addition, Pearson correlation and partial correlation tests were administered to determine the relationship among the variables included in this study. SPSS software was used for data analysis. Alpha level was set at .05 for all analysis.

\section{Results}

A Cronbach,s alpha of .670 was observed for Oldenburg burnout scale indicating sufficient level of internal consistency for this scale in the sample of university faculty in Pakistan.

Pearson correlation (2-tailed) was computed to determine the relationship of physical activity (total MET score), physical activity level (low, medium, high), and sedentary time with total score of burnout and burnout subscales of disengagement and exhaustion for 254 university faculty members.

The analysis yielded a significantly negative correlation of physical activity (total MET) with disengagement $(\mathrm{r}=-.132, \mathrm{~N}=254, \mathrm{p}<.05)$, exhaustion $(\mathrm{r}=-.160, \mathrm{~N}=254, \mathrm{p}<.05)$, and with total score of burnout $(\mathrm{r}=-.173, \mathrm{~N}=254, \mathrm{p}<.01)$. Physical activity level (low, medium, high) was significantly negatively correlated with total burnout score $(\mathrm{r}=-.155, \mathrm{~N}=254, \mathrm{p}<.05)$, exhaustion $(\mathrm{r}=-.143, \mathrm{~N}=$ $254, \mathrm{p}<.01)$ but not significantly related with disengagement $(\mathrm{r}=-.114, \mathrm{~N}=254, \mathrm{p}=.072)$. Sedentary time showed no significant relationship with total score of burnout $(\mathrm{r}=-.021, \mathrm{~N}=254, \mathrm{p}=.748)$, subscale of disengagement $(\mathrm{r}=-.023, \mathrm{~N}=254, \mathrm{p}=.724)$ and exhaustion $(\mathrm{r}=-.009, \mathrm{~N}=254, \mathrm{p}=.889)$.

Partial correlation was also computed while using age, gender, experience, and qualification as controlling variables to determine the relationship of total physical activity (MET score), sedentary time, and physical activity level with totals score on burnout measure, and subscales of disengagement and exhaustion. The analysis showed a significant negative correlation of total physical activity (Total MET) with total score of burnout $(\mathrm{r}=-.145, \mathrm{~N}=254, \mathrm{p}<.05)$ but showed no significant relationship with disengagement $(\mathrm{r}=-$ $.115, \mathrm{~N}=254, \mathrm{p}=.085)$ and exhaustion $(\mathrm{r}=-.126, \mathrm{~N}=254, \mathrm{p}=.058)$. Moreover, there was no significant correlation of physical activity level with burnout $(\mathrm{r}=-.103, \mathrm{~N}=254, \mathrm{p}=.124)$, disengagement $(\mathrm{r}=$ $.074, \mathrm{~N}=254, \mathrm{p}=.267)$ and exhaustion $(\mathrm{r}=-.097, \mathrm{~N}=254, \mathrm{p}=.147)$, respectively.

Moreover, Pearson correlation (2-tailed) analysis was performed to explore relationship of demographic variables including experience, designation, and ages on total score of physical activity, level of physical activity, total score of burnout scale, disengagement, and exhaustion. The analysis yielded a significant positive relationship of age with burnout total score, exhaustion but significantly negative association with total score of physical activity $(\mathrm{r}=-.181, \mathrm{~N}=254, \mathrm{p}<.01)$ and level of physical activity $(\mathrm{r}=-.191$, $\mathrm{N}=254, \mathrm{p}<.005)$ but no significant relationship with disengagement $(\mathrm{r}=.077, \mathrm{~N}=254, \mathrm{p}=.232)$ was found. Similarly, job experience was significantly negatively associated with total score of physical activity $(\mathrm{r}=-.127, \mathrm{~N}=254, \mathrm{p}<.05)$ and level of physical activity $(\mathrm{r}=-.224, \mathrm{~N}=254, \mathrm{p}<.001)$, positively related with total score of burnout $(\mathrm{r}=.078, \mathrm{~N}=254, \mathrm{p}<.01)$ and exhaustion $(\mathrm{r}=.223, \mathrm{~N}=$ $254, \mathrm{p}<.001)$ whereas no relationship with disengagement $(\mathrm{r}=.064, \mathrm{~N}=254, \mathrm{p}=.322)$ was observed. Moreover, there was significantly positive association of academic qualification with total burnout $(\mathrm{r}=$ $.141, \mathrm{~N}=254, \mathrm{p}<.05)$, exhaustion subscale $(\mathrm{r}=.128, \mathrm{~N}=254, \mathrm{p}<.05)$ and age $(\mathrm{r}=.342, \mathrm{~N}=254, \mathrm{p}<$ $.001)$. No significant relationship was observed of the designation with physical activity and burnout or any subscale of burnout.

\section{Discussions}

The study sought to determine relationship of physical activity with burnout among university professors. Findings indicated a significantly negative relationship of total score of physical activity with disengagement, exhaustion, and total burnout score. Physical activity level (low, medium, high) was 
significantly negatively correlated with total burnout score and subscale of exhaustion. The negative relationship remained evident between total physical activity and burnout after controlling for age, gender, experience, and academic qualification in partial correlation analysis but no significance relationships existed for disengagement and exhaustion. In general, the faculty members reported higher levels of physical activity tend to perceive less symptoms of burnout which indirectly lead towards better social functioning. This likely to suggest that physical activity contributes in reduction of burnout among the sample of university faculty in Pakistan. Physical activity and exercise can be used as a strategy to offset the negative effects of job related exhaustion and disengagement among university professors. Promoting physical exercise should be in preference to improve job related efficiency by improving symptoms of burnout in faculty members of higher education institutions.

The findings concerning positive effect of physical activity on faculty burnout observed in this study seems congruent with the findings of previous studies conducted by Moueleu Ngalagou et al. (2019); Sane et al. (2012) suggesting positive effect of sport and physical activates on burnout in university teachers. In line with these findings, other studies also provided support for the beneficial effects of physical activity on reducing emotional exhaustion (Carson et al., 2010) or decreased physical fitness as a result of decreased personal accomplishment (Carraro, Scarpa, Gobbi, Bertoll, \& Robazza, 2010) among school teachers. In the same line, a recent systematic review concluded that participation in physical exercise and sports activities can be considered an effective mean to prevent burnout(Naczenski, de Vries, van Hooff, \& Kompier, 2017).

The reasons of the result exploring negative relationship between physical activity and burnout are explicitly unknown. However, several explanations can be presented that might underlie the benefits of physical activity to reduce faculty burnout. For example, burnout characterized by psychological symptoms such as chronic stress, lack of vigor, mental fatigue, and depression (Herman et al., 2018; Sabagh et al., 2018) and physical activity reduces these symptoms (Penedo \& Dahn, 2005). In addition, physical activities contribute to enhance musculoskeletal fitness, strength, and cardiovascular fitness, reduce risk of diseases, improved physical health (Anderson \& Durstine, 2019) that might further contribute to prevent burnout.

The findings regarding age and job experience demonstrated that total score of burnout and subscale of exhaustion increase with increasing age but no effect on disengagement. However, age was inversely association with total score of physical activity and level of physical activity. Similar pattern of result was observed regarding relationship of job experience with physical activity and burnout. Academic qualification was positively related with total score of burnout, exhaustion, and age. These results collectively suggest that physical activity decreases but burnout increases with increasing age of the participants and the vice versa. This pattern of result suggests that younger and less experienced faculty members were more active and experiencing less burnout. As the age and experience increase; physical activity decreases and burnout increases. In contrast to results of this study, inverse relationship was reported for age and job experience on employee burnout by the study conducted by Brewer and Shapard (2004).

Possible reasons for this pattern of results might be that senior faculty members having higher qualification, experience, and obviously having greater ages thus may have some additional job related assignments, have more postgraduate level student, and higher engagement in research activities. Thus the multidimensional and multiple types of job related activities of senior faculty may require more physical and cognitive engagement, more switching between varieties of job assignments that tend to exert more physical and cognitive load. These might leave less room for doing physical exercise that possibly led to experience more burnout. These reasons logically enhance our understanding regarding the observed patterns of results mentioned above. It is likely that senior faculty more susceptible for the risk of appearance of burnout and sedentary behavior. These findings point to the suggestions that there should 
more focus on senior faculty members in regard to the interventions and programs to address the issues of physical activity and burnout.

In this connection, the interventions incorporating group based physical exercise programs for promoting health and well-being of university faculty that can be a useful approach to reduce or prevent burnout symptoms (Abós, Sevil-Serrano, Julián-Clemente, Generelo, \& García-González, 2019; Leininger, Adams, \& DeBeliso, 2015). For example, in a study, physical exercise programs that incorporated sports related activities, strength training and aerobic exercises were presented to secondary school teachers for the period of one academic year (Abós et al., 2019). This program found to be effective to improve vigor, work satisfaction, relatedness and absorption among teachers. Similar programs need to be launched on university campuses for faculty members to prevent or reduce burnout and to improve their social life.

\section{Limitations and future directions}

The sample for this study was selected from three public sector universities of southern Punjab. The sample would be more representative selecting from the universities situated in other geographical regions. In addition the selected sample included only public sector universities. Whereas the data from private sector universities is absent. Further studies need to overcome these limitations by focusing on both private and public sector universities from various geographical regions of the country. Another limitation of this study was that most of the sample consisted of junior faculty such as lecturers and assistant professors. There is need to consider senior faculty members such as associate professors and full professors. The design of this study was crass-sectional in nature that is less robust. There is need to address this issue using more stringer study designs including longitudinal, or randomized controlled designs to determine relationship between these variables. Although IPAQ short version considered robust with respect to validity and reliability, however, objective measures are suggested to use in future studied.

\section{Conclusion}

This study found that there is inverse relationship between physical activity and faculty burnout. This study further explored that there was positive relation of age with burnout symptoms, negative relation with physical activity. Similarly burnout was higher and physical activity was lower with increased teaching experience. These findings suggest buffering effect of physical activity on faculty burnout. Physical exercise suggested to be considered as interventional focus when dealing with problem of faculty burnout among public sector universities in Pakistan. These strategies should have more focus on faculty members with greater experience and age. It will be better for their social functioning too. Future studies are suggested using larger sample from private and public sector universities from various geographical regions to address these issues.

\section{References}

Abós, Á., Sevil-Serrano, J., Julián-Clemente, J. A., Generelo, E., \& García-González, L. (2019). Improving Teachers' Work-Related Outcomes through a Group-Based Physical Activity Intervention during Leisure-Time. The Journal of Experimental Education, 1-20. doi: 10.1080/00220973.2019.1681349

Ahmady, S., Changiz, T., Masiello, I., \& Brommels, M. (2007). Organizational role stress among medical school faculty members in Iran: dealing with role conflict. BMC Medical education, 7(1), 14.

Anderson, E., \& Durstine, J. L. (2019). Physical Activity, Exercise, and Chronic Diseases: A Brief Review. Sports Medicine and Health Science. doi: https://doi.org/10.1016/j.smhs.2019.08.006

Brewer, E. W., \& Shapard, L. (2004). Employee Burnout: A Meta-Analysis of the Relationship Between Age or Years of Experience. Human Resource Development Review, 3(2), 102-123. doi: $10.1177 / 1534484304263335$

Carraro, A., Scarpa, S., Gobbi, E., Bertoll, M., \& Robazza, C. (2010). Burnout and Self-Perceptions of Physical Fitness in a Sample of Italian Physical Education Teachers. Perceptual and Motor Skills, 111(3), 790-798. doi: 10.2466/06.07.11.14.pms.111.6.790-798 
Carson, R. L., Baumgartner, J. J., Matthews, R. A., \& Tsouloupas, C. N. (2010). Emotional Exhaustion, Absenteeism, and Turnover Intentions in Childcare Teachers: Examining the Impact of Physical Activity Behaviors. Journal of Health Psychology, 15(6), 905-914. doi: $10.1177 / 1359105309360697$

Committee, I. R. (2005). Guidelines for data processing and analysis of the International Physical Activity Questionnaire (IPAQ)-short and long forms. http://www. ipaq. ki. se/scoring. pdf.

Craig, C. L., Marshall, A. L., Sjöström, M., Bauman, A. E., Booth, M. L., Ainsworth, B. E., . . Sallis, J. F. (2003). International physical activity questionnaire: 12-country reliability and validity. Medicine \& Science in Sports \& Exercise, 35(8), 1381-1395.

Demerouti, E. (1999). Burnout: A consequence of specific working conditions among human service and production tasks. Frankfurt/Main: Lang.

Demerouti, E., \& Nachreiner, F. (1998). The specificity of burnout in human services: Fact or artifact? Zeitschrift fur Arbeitswissenschaft, 52(2), 82-89.

Dyrbye, L. N., Harper, W., Durning, S. J., Moutier, C., Thomas, M. R., Massie Jr, F. S., . . Sloan, J. A. (2011). Patterns of distress in US medical students. Medical teacher, 33(10), 834-839.

Dyrbye, L. N., Massie, F. S., Eacker, A., Harper, W., Power, D., Durning, S. J., . . Sloan, J. (2010). Relationship between burnout and professional conduct and attitudes among US medical students. JAMA, 304(11), 1173-1180.

El-Ibiary, S. Y., Yam, L., \& Lee, K. C. (2017). Assessment of burnout and associated risk factors among pharmacy practice faculty in the United States. American journal of pharmaceutical education, 81(4), 75.

Golub, J. S., Johns III, M. M., Weiss, P. S., Ramesh, A. K., \& Ossoff, R. H. (2008). Burnout in Academic Faculty of Otolaryngology-Head and Neck Surgery. The Laryngoscope, 118(11), 1951-1956. doi: 10.1097/MLG.0b013e31818226e9

González, K., Fuentes, J., \& Márquez, J. L. (2017). Physical inactivity, sedentary behavior and chronic diseases. Korean journal of family medicine, 38(3), 111.

Gonzalez, S., \& Bernard, H. (2006). Academic workload typologies and burnout among faculty in Seventh-day Adventist colleges and universities in North America. Journal of research on Christian education, 15(1), 13-37.

Herman, K. C., Hickmon-Rosa, J. e., \& Reinke, W. M. (2018). Empirically derived profiles of teacher stress, burnout, self-efficacy, and coping and associated student outcomes. Journal of Positive Behavior Interventions, 20(2), 90-100.

Hogan, R. L., \& McKnight, M. A. (2007). Exploring burnout among university online instructors: An initial investigation. The Internet and Higher Education, 10(2), 117-124.

Koutsimani, P., Anthony, M., \& Georganta, K. (2019). The relationship between burnout, depression and anxiety: A systematic review and meta-analysis. Frontiers in psychology, 10, 284.

Lackritz, J. R. (2004). Exploring burnout among university faculty: incidence, performance, and demographic issues. Teaching and Teacher Education, 20(7), 713-729.

Leininger, L. J., Adams, K. J., \& DeBeliso, M. (2015). Differences in health promotion program participation, barriers and physical activity among faculty, staff and administration at a university worksite. International Journal of Workplace Health Management, 8(4), 246-255.

May, R. W., Bauer, K. N., \& Fincham, F. D. (2015). School burnout: Diminished academic and cognitive performance. Learning and Individual Differences, 42, 126-131.

May, R. W., Sanchez-Gonzalez, M. A., Brown, P. C., Koutnik, A. P., \& Fincham, F. D. (2014). School burnout and cardiovascular functioning in young adult males: a hemodynamic perspective. Stress, 17(1), 79-87.

Moueleu Ngalagou, P. T., Assomo-Ndemba, P. B., Owona Manga, L. J., Owoundi Ebolo, H., Ayina Ayina, C. N., Lobe Tanga, M. Y., . . . Mandengue, S. H. (2019). Burnout syndrome and associated factors among university teaching staff in Cameroon: Effect of the practice of sport and physical

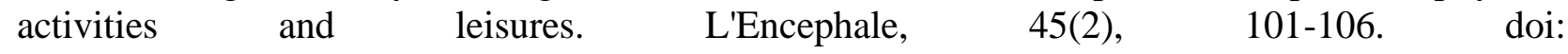
https://doi.org/10.1016/j.encep.2018.07.003

Naczenski, L. M., de Vries, J. D., van Hooff, M. L., \& Kompier, M. A. (2017). Systematic review of the 
association between physical activity and burnout. Journal of occupational health, 59(6), 477-494.

Organization, W. H. (2019). Global Health Observatory (GHO) data 2019, from https://www.who.int/gho/ncd/risk_factors/physical_activity_text/en/

Penedo, F. J., \& Dahn, J. R. (2005). Exercise and well-being: a review of mental and physical health benefits associated with physical activity. Current opinion in psychiatry, 18(2), 189-193.

Piercy, K. L., Troiano, R. P., Ballard, R. M., Carlson, S. A., Fulton, J. E., Galuska, D. A., . . Olson, R. D. (2018). The Physical Activity Guidelines for Americans. JAMA, 320(19), 2020-2028. doi: 10.1001/jama.2018.14854

Reis, D., Xanthopoulou, D., \& Tsaousis, I. (2015). Measuring job and academic burnout with the Oldenburg Burnout Inventory (OLBI): Factorial invariance across samples and countries. Burnout Research, 2(1), 8-18. doi: https://doi.org/10.1016/j.burn.2014.11.001

Sabagh, Z., Hall, N. C., \& Saroyan, A. (2018). Antecedents, correlates and consequences of faculty burnout. Educational Research, 60(2), 131-156. doi: 10.1080/00131881.2018.1461573

Samir, N., Mahmud, S., \& Khuwaja, A. K. (2011). Prevalence of physical inactivity and barriers to physical activity among obese attendants at a community health-care center in Karachi, Pakistan. BMC Research Notes, 4(1), 174. doi: 10.1186/1756-0500-4-174

Sane, M. A., Devin, H. F., Jafari, R., \& Zohoorian, Z. (2012). Relationship Between Physical Activity and It's Components with Burnout in Academic Members of Daregaz Universities. Procedia - Social and Behavioral Sciences, 46, 4291-4294. doi: https://doi.org/10.1016/j.sbspro.2012.06.242

Singh, R., Pattisapu, A., \& Emery, M. S. (2019). US Physical Activity Guidelines: Current state, impact and future directions. [Review]. Trends Cardiovasc Med, 17(19), 30140-30149.

Toker, S., \& Biron, M. (2012). Job burnout and depression: unraveling their temporal relationship and considering the role of physical activity. Journal of Applied Psychology, 97(3), 699.

Washington*, S., Quanstrom, K., Carroll, P., \& Greene, K. (2019). Mp15-08 The Interventions To Reduce Resident Exhaustion And Depersonalization (Tirred) Study: A Pilot Study Exploring Burnout In An Academic Urology Program. The Journal of Urology, 201(Supplement 4), e199e199.

Wright, J. G., Khetani, N., \& Stephens, D. (2011). Burnout among faculty physicians in an academic health science centre. Paediatrics \& child health, 16(7), 409-413. 